\title{
APLICAÇÃO EXÓGENA DE GA3 E TIAMETOXAM SOBRE A DINÂMICA DA GERMINAÇÃO DE SEMENTES DE Psidium guineense Swartz (MYRTACEAE)
}

\author{
Michele Aparecida dos Santos NOBREGA*, Montcharles da Silva PONTES \& Etenaldo Felipe SANTIAGO \\ Universidade Estadual de Mato Grosso do Sul. Dourados, Mato Grosso do Sul, Brasil. \\ *Autor para correspondência: nobrega_michele@yahoo.com.br
}

DOI: http://dx.doi.org/10.18571/acbm.173

\section{RESUMO}

Estudos com plantas nativas são de grande importância uma vez que auxiliam tanto na preservação das espécies quanto de seu habitat natural. Este trabalho teve como objetivo avaliar a influência do ácido giberélico e do tiametoxam na germinação de Psidium guineense. Foram realizados dois tratamentos com tiametoxam e dois com ácido giberélico bem como a associação da menor concentração de ambos, e um tratamento controle. Embora a aplicação da associação de $\mathrm{GA}_{3}$ e Tiametoxam tenha elevado em $27,7 \%$ o percentual germinativo, não foram verificadas diferenças significativas $(p<0,05)$ nas variáveis de percentual e de frequência relativa de germinação. Quanto ao tempo médio de germinação observou-se uma variação de $10,5 \%$. No entanto a velocidade de germinação foi similar entre os tratamentos. Observou-se apenas na maior concentração de Tiametoxam efeito significativo $(p<0,05)$ no coeficiente de variação do tempo. Avaliar os efeitos que diferentes substâncias bioestimuladoras possuem sobre a semente e a melhor forma de manuseá-los em espécies nativas propicia um melhor conhecimento da biologia das espécies.

Palavras-chave: Semente; Espécie nativa; Fitoestimulante; Araçá.

\begin{abstract}
Studies with native plants are of great importance since they assist both the preservation of the species of your natural habitat. This work aimed to evaluate the influence of gibberellic acid and tiametoxam on germination of Psidium guineense. Two treatments were carried out with tiametoxam and two with gibberellic acid as well as the Association of lower concentration of both, and a control treatment. Although the application of GA3 and Tiametoxam Association has $27.7 \%$ high percentage were not germinative noted significant differences $(\mathrm{p}<0.05)$ in the variables of relative frequency and percentage of germination. As for the average germination was observed an increase of $10.5 \%$. However the speed of germination was similar among treatments. It was observed only at the highest concentration of Tiametoxam significant effect $(p<0.05)$ the coefficient of variation of the time. Evaluate the effects of different bioestimuladoras substances have on the seed and the best way of handling them in native species provides a better understanding of the biology of the species.
\end{abstract}

Keywords: Seed; Native species; Fitoestimulante; Araçá.

\section{Introdução}

O histórico de uso e ocupação dos solos brasileiros seguiu o modelo de desenvolvimento agrícola no qual a degradação de recursos e a redução da biodiversidade foram preponderantes (ALMEIDA, 2000; SANTIAGO \& PAOLI, 2007), a falta de planejamento no uso dos recursos naturais tem resultado na degradação dos ecossistemas, sendo relevante destacar que a cobertura vegetal, que originalmente ocupava a maior parte do território brasileiro, vem historicamente 
cedendo espaço para atividades antrópicas (SCALON et al., 2009; NOBREGA et al. 2014; SANTIAGO et al. 2015).

A recuperação da cobertura vegetal nativa é interdependente dos estudos do comportamento das espécies, tal correlação gerou uma grande necessidade de aumentar o acervo biológico sobre seu comportamento a fim de serem utilizadas em processos de reposição florestal. Neste sentido, a execução de trabalhos que enfocam espécies ocorrentes no Cerrado é fundamental para dar suporte tanto às práticas conservacionistas como de uso sustentável de seus recursos (LEHN et al., 2008).

O conhecimento do processo germinativo bem como de substâncias capazes de aperfeiçoálo é de grande relevância nos estudos botânicos, em especial no campo da produção de mudas, assumindo maior importância dada à diversidade dos ecossistemas tropicais. A despeito da riqueza vegetal, dados sobre testes de propagação e produção de mudas com espécies nativas do Cerrado são ainda insuficientes (SCALON et al., 2009).

O conhecimento dos fatores capazes de influenciar a germinação das sementes contribui para aperfeiçoar o manejo das espécies nativas do bioma, pois este processo é altamente influenciado por fatores ambientais, como incidência de luz, temperatura e substrato; e fatores fisiológicos como concentração de hormônios. Estes fatores podem ser manipulados a fim de analisar que resultados podem ser obtidos devido a essas determinadas condições (GELORMINI 1995; NASSIF et al., 2004; BOUCELHA \& DJEBBAR 2015).

Psidium guineense Swartz (Araçá), é uma arvoreta de até $6 \mathrm{~m}$ de altura, distribuída em quase todos os estados do Brasil, existindo relatos de ocorrência do Rio Grande do Sul até o Amazonas. Apresentam ampla distribuição ocorrendo nas restingas, tabuleiros, cerradões e capoeiras (BRANDÃO et al., 2002. BEZERRA et al., 2006).

Segundo Leonel \& Pedroso (2005), o emprego de reguladores de crescimento sobre a germinação e o crescimento das mudas de espécies frutíferas, de modo isolado, já vêm sendo desenvolvidos a algum tempo e, atualmente, em associação com outros reguladores e com o emprego de bioestimulantes (FERREIRA et al., 2007). Devido ao seu grande potencial na área comercial e agronômica, trabalhos relacionados à influência do ácido giberélico $\left(\mathrm{GA}_{3}\right)$ em plantas têm aumentado significativamente nas últimas décadas (REEVE \& CROZIER, 1974; FERREIRA et al., 2002; MATTANA et al., 2012).

Assim como o $\mathrm{GA}_{3}$, o tiametoxam (3-(2-cloro-1,3-tiazol-5-imetil)-5-metil-1,3,5oxadiazinam-4-idenem (nitro) amina) pode melhorar o processo germinativo devido a sua atuação na ativação de enzimas metabólicas, sendo ambos caracterizados como biotivadores. Os bioativadores são substâncias naturais de origem vegetal que possuem ações semelhantes aos principais reguladores vegetais, visando o crescimento e o desenvolvimento da planta. Proporcionam um melhor equilíbrio fisiológico, favorecendo uma maior aproximação ao potencial genético da cultura (CASTRO, 2006).

As giberelinas constituem uma classe de hormônios capaz de modular o desenvolvimento durante todo o ciclo de vida da planta, na maioria das vezes, o seu componente bioativo é o GA3. Esta classe de hormônio é conhecida por atuar no alongamento caulinar, promover a germinação em algumas espécies, diferenciação foliar, controle do meristema apical caulinar, transição da fase juvenil para a madura, determinação sexual, iniciação e desenvolvimento floral, iniciação e desenvolvimento dos frutos (KERBAUY, 2008). Operando também no controle de hidrólise das reservas, pela indução da síntese da $\alpha$-amilase, enzima responsável pela hidrólise do amido (MAZLIAK, 1998). Já o tiametoxam, é um inseticida sistêmico do grupo neonicotinóide, tem efeito bioativador, atuando na expressão dos genes responsáveis pela síntese e ativação de enzimas metabólicas, relacionadas ao crescimento da planta, alterando a produção de aminoácidos precursores de hormônios vegetais (CASTRO, 2006; CASTRO et al. 2008).

Apesar da importância desses compostos, não foram encontrados na literatura dados relacionados ao efeito dos mesmos na germinação de sementes de P. guineense Swartz (Araçá). 
Desse modo, torna-se importante, estudos que abordem o efeito desses fitorreguladores na germinação de sementes dessa espécie.

Este trabalho teve como objetivo avaliar a germinação de sementes de P. guineense Swartz submetidas à aplicação exógena de $\mathrm{GA}_{3}$ e tiametoxam como forma de conhecer os efeitos destes compostos sobre o comportamento germinativo, contribuindo com a obtenção de dados úteis ao manejo desta espécie.

\section{Materiais e Métodos}

\subsection{Material Botânico}

O material botânico consistiu de frutos de Psidium guineense Swarts (Araçá), obtidos de matrizes presentes na Universidade Estadual de Mato Grosso do Sul - UEMS - Unidade de Dourados. Os frutos foram levados para o Laboratório de Ecologia - Centro de Análise e Monitoramento Ambiental (LE-CInAM) para o beneficiamento manual com auxílio de peneira. Descartaram-se as sementes aparentemente danificadas ou chochas e efetuou-se a secagem prévia das demais sementes em bandejas plásticas forradas com papel de filtro, sendo estas prontamente utilizadas, sem armazenamento prévio.

\subsection{Tratamento}

Foram efetuados seis tratamentos distintos: Tratamento 1: Controle (C); Tratamento 2: dose 1 de $\mathrm{GA}_{3}\left(0,025 \mathrm{mg} / \mathrm{mL}\right.$ de $\left.\mathrm{GA}_{3}\right)$; Tratamento 3: dose 2 de $\mathrm{GA}_{3}\left(1,0 \mathrm{mg} / \mathrm{mL}\right.$ de $\left.\mathrm{GA}_{3}\right)$; Tratamento 4: dose 1 de Tiametoxam $(0,25 \mathrm{ml}$, equivalente a $0,06 \%$ de tiametoxam); Tratamento 5: dose 2 de Tiametoxam ( $5 \mathrm{~mL}$ que equivale a 10\%); Tratamento 6: união da menor dosagem de ácido giberélico (T2) com a menor dosagem de Tiametoxam (T4). As sementes utilizadas foram imersas nas soluções por vinte e quatro horas sendo posteriormente lavadas com o auxilio de uma peneira Granutest $0,5 \mathrm{~mm}$ e dispostas em placas de petri forradas com duas folhas de papel filtro, sendo empregado para cada parcela experimental cinco repetições com 25 sementes cada, totalizando 250 sementes por tratamento.

\subsection{Germinação}

Os testes de germinação foram conduzidos em câmara de germinação do tipo BOD, regulada a $25 \pm 2{ }^{\circ} \mathrm{C}$ mantidos sob luz branca fluorescente contínua $\left(21,8 \pm 5,7 \mu \mathrm{mol} \mathrm{m}^{-2} \mathrm{~s}^{-1}\right)$ durante um período de 30 dias. Foram realizadas avaliações dias, considerando como critério de germinação a emissão da raiz primária.

Foram avaliados os parâmetros de percentual germinativo e o tempo médio de germinação de acordo com Labouriau (1983), o índice de velocidade de germinação (MAGUIRE, 1962) e a variação da germinação no tempo (SANTANA \& RANAL, 2004), a distribuição da frequência relativa de germinação e a incerteza $(U)$ associada à frequência, calculadas de acordo com Labourial \& Valadares (1976), coeficiente de variação do tempo (SANTANA \& RANAL, 2004) e o índice de sincronização (Z) adaptado de Primack (1980).

\subsection{Análises Estatísticas}

O delineamento experimental utilizado foi o inteiramente casualizado. Os dados obtidos foram submetidos a análise de variância ANOVA. Os dados de porcentagem de germinação considerando o fitoestimulante de maior efeito, foram submetidos à análise de regressão polinomial. O modelo da regressão foi determinado com base na lógica fisiológica da germinação, na significância dos coeficientes de regressão realizada pelo teste $\mathrm{t}$ de Student a $5 \%$ de 
probabilidade e nos valores do coeficiente de determinação. Os gráficos foram confeccionados, utilizando-se o software SciDAVis 1.D5.

\section{Resultados}

Não foram encontradas diferenças significativas na germinação, tempo médio, velocidade, percentual germinativo, frequência relativa e variação da germinação no tempo (Tabela 1) para sementes tratadas com $\mathrm{GA}_{3}$, tiametoxam ou a interação de ambos nas concentrações utilizadas neste trabalho, embora no tratamento $\mathrm{GA}_{3}(2)$ o tempo médio tenha sido $10,5 \%$ superior quando comparado ao controle, essa diferença não foi significativa pela análise de variância (tendo o valor de $\alpha=0,05)$, com relação ao coeficiente de variação do tempo apenas a combinação $\mathrm{GA}_{3}(1)+\mathrm{Tx}$ (1) apresentou diferença significativa com o controle.

Considerando a cinética da germinação, observou-se maior homogeneidade temporal com diferença significativa $(\mathrm{p}<0,05)$ no tratamento $\mathrm{Tx}(2)(\mathrm{CVt}=15,57)$ e a maior heterogeneidade temporal foi observada na combinação $\mathrm{GA}_{3}(1)+\mathrm{Tx}(1),(\mathrm{CVt}=19,50)$. Quanto à sincronização da germinação o tratamento $\mathrm{GA}_{3}(1)$ foi responsável pela maior sincronia $(Z=0,06)$ e Tx (2) apresentou menor sincronia $(Z=0,09)$, sugerindo efeito na heterogeneidade fisiológica das sementes de $P$. guineense Swartz em resposta a maior dose de Tiametoxam, mostrando perda de sincronia na germinação.

Tabela 1. Velocidade media $(v)$, porcentagem de germinatção $(\% G)$, tempo médio de germinação $(t)$, percentual germinativo $(\% G)$, Indice de velocidade de germinação $(I V G)$, coeficiente de variação da germinação no tempo $(C V t)$, incerteza associada a frequência $(\mathrm{U})$ e sincronia da germinação $(Z)$ nos diferentes tratamentos.

\begin{tabular}{cccccccc}
\hline & $\mathbf{v}$ & $\mathbf{t}$ & $\mathbf{\% G}$ & $\mathbf{G V I}$ & $\mathbf{C V t}$ & $\mathbf{U}$ & $\mathbf{Z}$ \\
\hline Controle & $0,05^{\mathrm{a}}$ & $17,42^{\mathrm{a}}$ & $58.4^{\mathrm{a}}$ & $0.8^{\mathrm{a}}$ & $19^{\mathrm{a}}$ & 3,5798 & 0,090326 \\
$\mathbf{G A 3}(\mathbf{1})$ & $0,05^{\mathrm{a}}$ & $17,79^{\mathrm{a}}$ & $71.6^{\mathrm{a}}$ & $0.9^{\mathrm{a}}$ & $19,40^{\mathrm{a}}$ & 2,34878 & 0,088674 \\
$\mathbf{G A 3}(\mathbf{2})$ & $0,05^{\mathrm{a}}$ & $19,82^{\mathrm{a}}$ & $75.2^{\mathrm{a}}$ & $0.88^{\mathrm{a}}$ & $18.50^{\mathrm{a}}$ & 2,51546 & 0,078638 \\
Tx (1) & $0,05^{\mathrm{a}}$ & $19,14^{\mathrm{a}}$ & $72^{\mathrm{a}}$ & $0.87^{\mathrm{a}}$ & $17,97^{\mathrm{a}}$ & 2,58317 & 0,076383 \\
Tx (2) & $0,05^{\mathrm{a}}$ & $17,42^{\mathrm{a}}$ & $57.6^{\mathrm{a}}$ & $0.82^{\mathrm{a}}$ & $15,57^{\mathrm{b}}$ & 2,52631 & 0,097135 \\
$\mathbf{G A 3}(\mathbf{1})+\mathbf{T x}(\mathbf{1})$ & $0,05^{\mathrm{a}}$ & $18,2^{\mathrm{a}}$ & $86.15^{\mathrm{a}}$ & $0.86^{\mathrm{a}}$ & $19,50^{\mathrm{a}}$ & 2,41388 & 0,06875 \\
\hline
\end{tabular}

Médias seguidas de mesma letra em linhas não diferem estatisticamente entre si ao nível de significância 5\% pelo teste de Tukey.

As doses de $\mathrm{GA}_{3}$ e Tiametoxam utilizadas nesta espécie, não foram suficientes para promover alguma alteração nos dados mensurados, sugerindo que as concentrações utilizadas não foram suficientes para promover alguma grande alteração na porcentagem germinação das sementes. Entretanto, no tratamento $\mathrm{GA}_{3}$ (2), o tempo médio foi $10,5 \%$ superior quando comparado ao controle, embora sugira que o aumento nas concentrações possa ocasionar um efeito negativo, aumentando o tempo médio de germinação, este resultado deve ser interpretado com cautela, pois, hormônios vegetais em excesso podem inibir a germinação e o desenvolvimento na planta. De maneira semelhante, o aumento de $27,75 \%$ no percentual germinativo obtido na combinação GA 3 (1) + Tx (1), também deve ser analisado com cautela, sugerindo a necessidade de novos estudos inclusive com outras espécies a respeito desta interação.

As curvas acumulativas de germinação (Figura 1. A) tanto para o controle como ambos os tratamentos se ajustam ao modelo não-linear logístico, que retratou melhor o comportamento germinativo das sementes, corroborando o comportamento longitudinal, matematicamente caracterizado como sigmiode, tendo função crescente nos estágios iniciais de desenvolvimento, tendo posteriormente um aumento de menor intensidade, que chega a estabilidade ao longo do tempo. A frequência relativa da germinação esteve distribuída entre o décimo e vigésimo dia, com vários picos germinativos, com maiores valores de frequência em torno do vigésimo dia (Figura 1. B), valendo ressaltar que esta variável apresentou um comportamento polimodal. 

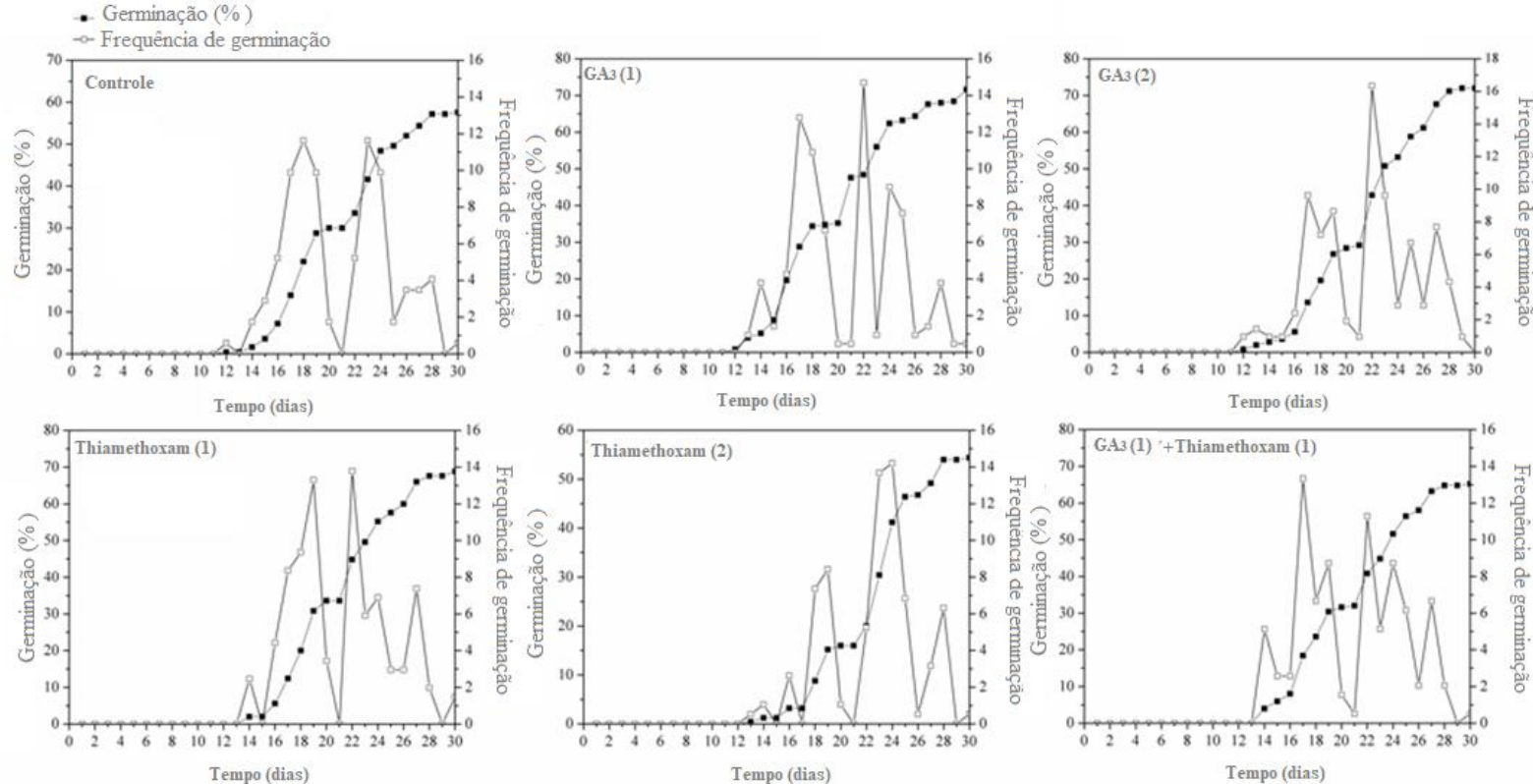

Figura 1: Percentual acumulativo e Frequência Relativa da germinação de sementes de Psidium guineense tratadas com ácido giberélico e tiametoxam: C- controle; $\mathrm{GA}_{3}(1)$ - 0,025 mg/mL de $\mathrm{GA}_{3} ; \mathrm{GA}_{3}(2)-1,0 \mathrm{mg} / \mathrm{mL}$ de $\mathrm{GA} 3$; $\mathrm{Tx}$

(1)- 0,25 ml de tiametoxam; Tx (2)- $5 \mathrm{~mL}$ de tiametoxam; $\mathrm{GA}_{3}(1)+\mathrm{Tx}(1)-0,025 \mathrm{mg} / \mathrm{mL}$ de $\mathrm{GA}_{3}+0,25 \mathrm{ml} \mathrm{de}$ tiametoxam.

Avaliando o uso do Tiametoxam, e, a interação com $\mathrm{GA}_{3}$ (Fig 2), observou-se ajuste quadrático com $\mathrm{R}^{2}=0,53$. Este resultado sugere que, se de maneira isolada, Tx e GA 3 não afetaram de maneira significativa a germinação das sementes de Psidium guineense, entretanto, o uso integrado desses dois bioestimulantes, com variação de suas dosagens sugere incremento na germinação.
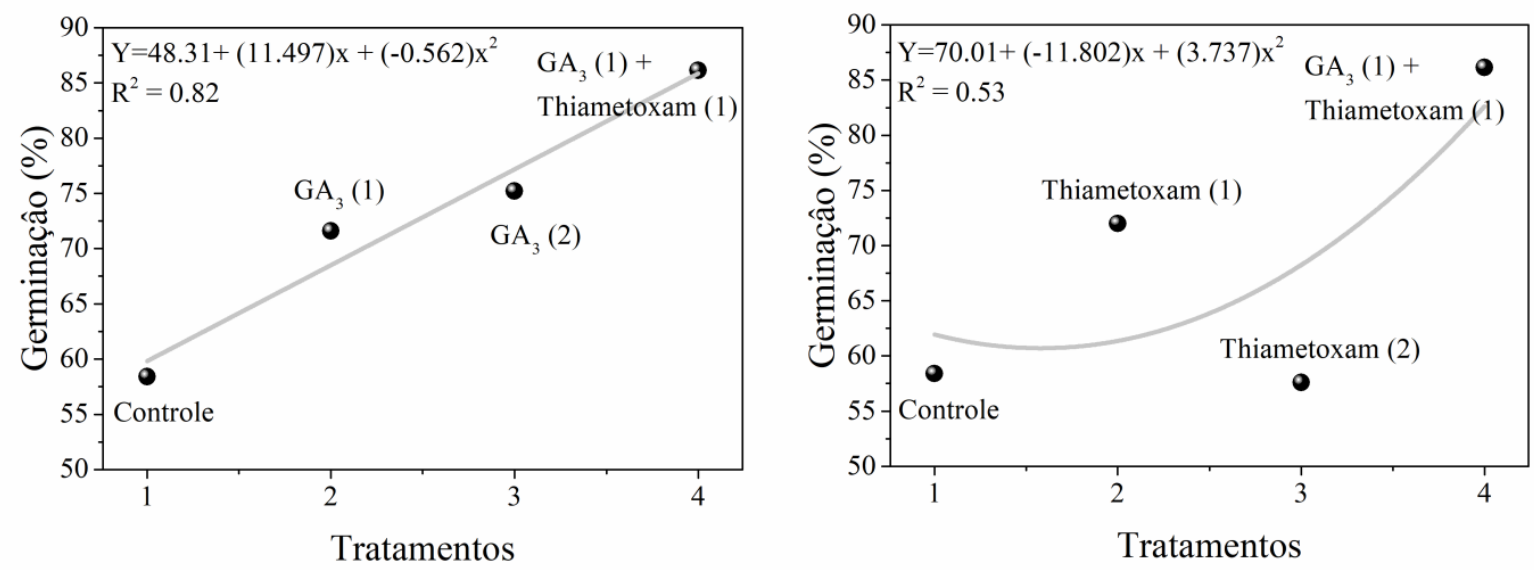

Figura 2: Ajuste quadrático para o Percentual Germinativo de sementes de Psidium guineense tratadas com diferentes doses de Ácido Giberélico $\left(\mathrm{GA}_{3}\right)$ e Tiametoxam (Tx): 1 - Controle; 2 - Tx 0,25 ml; 3 - Tx 5mL; 4 - GA 3 $0,025 \mathrm{mg} / \mathrm{mL}+\mathrm{Tx} 0,25 \mathrm{ml}(0,06 \%)$.

\section{Discussão}

Estes resultados sugerem que as concentrações de hormônio dentro da semente, sejam suficientes para que esta realize seu processo germinativo. Em seu trabalho, Zamith \& Scarano (2004), estudando espécies de Psidium, obtiveram um percentual máximo germinativo de 70\%, resultados similares foram observados neste trabalho, que apresentou percentual máximo germinativo de 75,2 \%. Estudando a germinação de sementes de porta-enxertos cítricos, Sousa et al., (2002), também não verificaram a promoção da germinação pelo GA 3 e atribuíram este 
resultado a um adequado nível endógeno de giberelina nas sementes, de forma que a adição do $\mathrm{GA}_{3}$ não interferiu em sua performance durante a germinação.

As concentrações de $\mathrm{GA}_{3}$ e Tiametoxam utilizadas neste estudo, não foram capazes de promover incremento significativo na germinação de sementes de $P$. guineense Swartz, tal resultado corrobora com os que foram observados por Tavares et al., (2007) nos quais constataram a ausência de interferências positivas ou negativas para o potencial de germinação das sementes de soja tratadas com Tiametoxam.

Não foram observadas diferenças significativas entre os tratamentos na velocidade média de germinação, tais dados corroboram com os de Ferreira et al., (2002), que estudando frutas do conde (Annona squamosa L.) tratadas com $\mathrm{GA}_{3}$ e não encontrou diferenças significativas para esta variável. A cinética temporal da germinação foi afetada significativamente, apenas quanto ao coeficiente de variação do tempo $(\mathrm{CVt})$ o que indica maior homogeneidade na distribuição da germinação ao longo do tempo em resposta ao Tiametoxam (Tx (2)).

Da mesma forma que o tempo médio, percentual germinativo, velocidade média, variação no tempo são importantes no manejo de sementes, a frequência relativa serve para indicar respostas fisiológicas de sementes de espécies nativas (FERREIRA et al., 2001). Esta variável apresentou um comportamento polimodal, tal comportamento é comum em espécies nativas, pois, permanecem fotodormentes no interior das matas enquanto a comunidade vegetal permanece intacta, a partir do momento em que a terra é remexida, ou quando se abre uma clareira ou na mata, ou ainda quando são transportadas para fora daquele ambiente, esse estado de fotodormência é quebrado, tal comportamento proporciona a formação de um "banco de sementes" no local em que se encontram (OLIVEIRA, 2007).

O caráter polimodal de germinação das sementes de $P$. guineense sugere que, sob condições naturais, a germinação pode se estender de dias a meses, desde que as sementes se mantenham viáveis no substrato em que se encontram, e indica também que a espécie tende a estabelecer bancos de sementes persistentes, isto é, com germinação espaçada no tempo. Para produção de mudas florestais, seja para fins de comerciais, restauração e repovoamento de cobertura vegetal nativa em áreas degradadas ou plantios heterogenios em agroflorestas, a obtenção de mudas de espécies nativas num mesmo período, com comportamento germinativo concentrado e deslocado para a esquerda da moda nos polígonos de frequência, implica num maior controle da produção, resultando na redução de custos e otimização do processo desde que haja qualidade fisiológica nas plântulas (PONTES et al., 2014).

Do ponto de vista ecológico este comportamento é de grande importância, pois além de garantir que a sementes germinem em boas condições possibilita também a regeneração natural das matas quando ocorre a abertura de clareiras (ALMEIDA, 2000). No entanto, não foi encontrado na literatura dados que afirmem que essa espécie apresente dormência. $\mathrm{O} \mathrm{GA}_{3}$ não parece estar envolvido no controle da dormência por si só, mas sim é importante na promoção e manutenção da germinação.

Estes resultados sugerem que, de maneira isolada, tanto o $\mathrm{GA}_{3}$, quanto o Tiametoxam, não influíram de maneira significativa a germinação das sementes de Psidium guineense, entretanto, o uso integrado desses dois bioestimulantes, nos parece promissor e expressa à carência na literatura de estudos inter-relacionando o uso do $\mathrm{GA}_{3}$ e do Tiametoxam em ação conjunta, sobre o comportamento germinativo de sementes de espécies nativas, sobretudo, espécies de cerrado como P. guineense Swartz.

\section{Conclusão}

A utilização de $\mathrm{GA}_{3}$ e Tx em doses isoladas não promoveram alterações significativas na germinação de sementes de Psidium guineense Swartz recém-colhidas, sendo necessária a realização de estudos com sementes armazenadas utilizando a interação entre diferentes doses 
destes produtos, o que pode contribuir ainda mais ao estudo do processo germinativo de espécies nativas.

Os resultados obtidos, e em especial os altos índices germinativos, superiores a $50 \%$ mesmo no controle, não indicam necessariamente, que as sementes desta espécie sejam indiferentes aos tratamentos, mas sim que possuem uma concentração de hormônio suficiente para realizar o processo germinativo, de modo que a quantidade de fitoestimulante utilizada em dose isolada não foi suficiente para gerar efeitos positivos e/ou negativos.

$\mathrm{O}$ efeito de diversos biorreguladores sobre os aspectos germinativos de sementes de espécies nativas requerem maiores esforços de pesquisa, tanto pela escassez de dados como, também por propiciar um melhor conhecimento de como manuseá-las, contribuindo para um maior conhecimento da biologia destas espécies.

\section{Referências}

ALMEIDA D.S., 2000. Recuperação ambiental da Mata Atlântica. Ilhéus: Editus. 300p.

BEZERRA J.E.F., et al. Cap. 3 - Araçá. Frutas nativas da região Centro-Oeste /Roberto Fontes Vieira... [et al.] (editores). -- Brasília: Embrapa Recursos Genéticos e Biotecnologia, 2006.

BOUCELHA L., \& DJEBBAR R., 2015. Influence de différents traitements de prégermination des graines de Vigna unguiculata (L.) Walp. sur les performances germinatives et la tolérance au stress hydrique. Biotechnol. Agron. Soc. Environ., 19, 160-172.

BRANDÃO M., et al., 2002. Árvores nativas e exóticas do Estado de Minas Gerais. Belo Horizonte: EPAMIG. 528 p

CASTRO P.R.C., 2006. Agroquímicos de controle hormonal na agricultura tropical. Piracicaba: ESALQ, 2006. 46p. (Série Produtor Rural, 32).

CASTRO G.S.A., et al., 2008. Tratamento de sementes de soja com inseticidas e um bioestimulante. Pesq. Agrop. Bras., 43, 1311-1318.

FERREIRA G. et al., 2001. Germinação de sementes de Asteraceae nativas no Rio Grande do Sul, Brasil. Acta Bot. Bras., 15(2), 231-242.

FERREIRA G., SEIDEL G.O., \& VERONA, M. M., 2001. Efeito de fitorreguladores na germinação de sementes de Atemóia (Annona cherimola Mill. X Annona squamosa L.).In: Anais do VIII Congresso Nacional de Fisiologia Vegetal, Ilhéus, BA.

FERREIRA G. et al., 2002. Uso de ácido giberélico em sementes de fruta-do-conde (Annona squamosa L.) visando à produção de mudas em diferentes embalagens. Rev. Bras. Frut., 24, 178182.

FERREIRA G. et al., 2007. Emergência e desenvolvimento de plântulas de maracujazeiro azedo oriundas de sementes tratadas com bioestimulante. Rev. Bras. Frut., 29,.595-599.

GELORMINI G., 1995. Optimisation des propriétés germinatives des graines de colza par initialisation : aspects méthodologiques et fondamentaux. Thèse de doctorat : Université de Rennes (France). 
KERBAUY G.B., 2008. Fisiologia Vegetal. 2a edição - Rio de Janeiro: Ed. Guanabara Koogan.

LABOURIAU L.G., 1983. A germinação das sementes. Secretaria geral da OEA Washington.

LABOURIAU L.G., \& VALADARES M.E.B., 1976. On the germination of seeds of Colotropis procera (Ait.) Ait. F. Anal. Bras. Acad. Ciên., 48, 263-284.

LEONEL S., \& PEDROSO C.J., 2005. Produção de mudas de maracujazeiro-doce com o uso de biorregulador. Rev. Bras. Frut., 27, 107-109.

LEHN C.R., ALVES F.M., \& DAMASCENO JÚNIOR G.A., 2008. Florística e fitossociologia de uma área de Cerrado Sensu stricto na região da borda oeste do Pantanal, Corumbá, MS, Brasil. Pesq. Bot., 59, 129-142.

MAGUIRE J.D., 1962. Speed of germination-aid in selection and evaluation for seedling emergence and vigor. Crop Sci., 2, 176-177.

MATTAMA E. et al., 2012. Interchangeable effects of gibberellic acid and temperature on embryo growth, seed germination and epicotyl emergence in Ribes multiflorum ssp. sandalioticum (Grossulariaceae). Plant Biol., 14, 77-87.

MAZLIAK P., 1998. Physiologie végétale. 2. Croissance et développement. Paris : Hermann éditeurs.

NASSIF S.M.L. et al. 2004. Fatores externos (ambientais) que influenciam na germinação de sementes. Disponível em: < http://www.ipef .br/tecsementes/germinacao.html>

NOBREGA M.A.S. et al., 2014. Impactos Ambientais Perceptíveis em Ecossistemas Urbanos e os Índices de Qualidadede da Água Ensaios e Ciência: Ciênc. Bio. Agrá. Saúde. v. 18, n. 4, p. 195-203.

OLIVEIRA S.F., 2007. Comparação do banco de sementes do solo de três fitofisionomias do bioma cerrado em áreas perturbadas. Dissertação (Mestrado em Ciências Florestais)-Universidade de Brasília, Brasília.

PONTES M.S. et al., 2014. Germinação de sementes de Maclura tinctoria (L.) D. Dom ex Steud. (Moraceae) embebidas em lodo de esgoto. Cad. Agroecol., 9, 1-8.

PRIMACK R.B., 1980. Variation in the phenology of natural populations of montane shrubs in New Zeland. J Ecolo., 68, 849-862.

REEVE D.R., \& CROZIER A., 1974. An assessment of gibberellin structure-activity relationships. J. Exp. Bot., 25, 431-445.

SANTANA D.G., \& RANAL M.A., 2004.Análise da germinação: um enfoque estatístico. Editora universidade de Brasilia. 247p.

SANTIAGO, E.F., PAOLI, A.A.S. Morphological responses in Guibourtia hymenifolia (Moric.) J. Leonard (Fabaceae) and Genipa americana L. (Rubiaceae) to nutrient deficit and flooding stress. Braz. J. Bot. 30, 131-140. 2007 
SANTIAGO E.F. et al., 2015. Can the Chlorophyll-a fluorescence be useful in identifying acclimated young plants from two populations of Cecropia pachystachya Trec. (Urticaceae), under elevated CO2 concentrations? J Fluoresc. 25, 49-57.

SCALON S.P.Q. et al., 2009. Germinação de sementes e crescimento inicial de mudas de Campomanesia adamantiumcamb.: efeito da lavagem, temperatura e de bioestimulantes. Rev. Bras.Sem., 31, 096-103.

SOUSA H.U. et al., 2002. Efeito do ácido giberélico sobre a germinação de sementes de porta enxertos cítricos. Rev. Bras. Frut., 24, 496-499.

TAVARES S. et al., 2007. Avaliação dos efeitos fisiológicos de thiametoxan no tratamento de sementes de soja. Rev. de Agric., 82, 47-54.

ZAMITH L.R., \& SCARANO F.R., 2004. Produção de mudas de espécies das Restingas do município do Rio de Janeiro, RJ, Brasil. Acta Bot. Bras., 18, 161-176. 